\title{
The product of the mouse nude locus, Whn, regulates the balance between epithelial cell growth and differentiation
}

\author{
Janice L. Brissette, ${ }^{1}$ Jie Li, ${ }^{1}$ Jun Kamimura, ${ }^{1,2}$ David Lee, ${ }^{1}$ and G. Paolo Dotto ${ }^{1,3}$ \\ ${ }^{1}$ Cutaneous Biology Research Center, Massachusetts General Hospital and Harvard Medical School, Charlestown, \\ Massachusetts 02129 USA, and ${ }^{2}$ Pharmaco Science Research Laboratories, Shiseido Research Center, Yokohama, 223, Japan
}

\begin{abstract}
Mutations in the winged-helix nude (whn) gene result in the nude mouse and rat phenotypes. The pleiotropic nude phenotype which affects the hair, skin, and thymus suggests that whn plays a pivotal role in the development and/or maintenance of these organs. However, little is known about whn function in these organs. We show here that in skin whn is specifically expressed in epithelial cells and not the mesenchymal cells, and using a hair reconstitution assay, we demonstrate that the abnormal nude mouse hair development is attributable to a functional defect of the epithelial cells. Examination of nude mouse primary keratinocytes in culture revealed that these cells have an increased propensity to differentiate in an abnormal fashion, even under conditions that promote proliferation. Furthermore, nude mouse keratinocytes displayed a 100-fold increased sensitivity to the growth-inhibitory/differentiation effects of the phorbol ester TPA. In parallel with these findings, we directly show that whn functions as a transcription factor that can specifically suppress expression of differentiation/TPA-responsive genes. The region of Whn responsible for these effects was mapped to the carboxy-terminal transactivating domain. These results establish whn as a key regulatory factor involved in maintaining the balance between keratinocyte growth and differentiation. The general implications of these findings for an epithelial self-renewal model will be discussed.
\end{abstract}

[Key Words: Whn; transcriptional factor; nude mouse; keratinocytes]

Received June 3, 1996; revised version accepted July 12, 1996.

The skin and its appendage, the hair follicle, provide excellent models for the study of the complex control mechanisms involved in organ morphogenesis and homeostasis. The formation and maintenance of the epidermis and the hair follicle, both self-renewing structures, require a balance between keratinocyte growth and differentiation. This coordinated control is accomplished through a complex network of intra- and intercellular signals that function both within the epithelial layers and between the epidermis and underlying dermal mesenchyme. Intracellular signaling pathways controlling the switch between keratinocyte growth and differentiation involve the activation of specific tyrosine kinases (Calautti et al. 1995, and references therein), phospholipase C (Moscat et al. 1989), and protein kinase C isoforms (Osada et al. 1993; Denning et al. 1995). Intercellular signaling mechanisms are best illustrated during hair follicle development, where a series of dermal-epidermal signaling events culminates with the mesenchymal cells of the dermal papilla inducing the epithelial cells of the hair plug to grow and differentiate into a

${ }^{3}$ Corresponding author. follicle (Hardy 1992). The nature of these intercellular signaling events and how they influence the intracellular control mechanisms remain largely unknown.

The spontaneously occurring nude mouse mutation has pleiotropic effects that result in the abnormal development of the skin, hair follicles, and thymus (Flanagan 1966; Pantelouris 1973). Although the nude mouse appears hairless, its dermis contains the same number of hair follicles as a wild-type mouse, but these follicles are aberrant and incompletely developed (Flanagan 1966; Köpf-Maier et al. 1990). The abnormal keratinization of the hair follicles is characterized by short, bent hair shafts that rarely protrude from the follicles. Keratinization of the epidermis is also aberrant in that the stratum corneum contains highly irregular piles of cornified debris. These animals also have a rudimentary thymus, and as a result have a severely impaired immune system because of the absence of $\mathrm{T}$ lymphocytes (Pantelouris 1973).

The mutated gene responsible for the nude mouse and rat phenotypes was identified recently. The gene, designated winged-helix nude $(w h n)$, encodes a putative transcription factor belonging to the winged-helix domain family (Nehls et al. 1994; Segre et al. 1995). Winged-helix proteins are regulated developmentally and direct tissuespecific transcription and cell fate decisions (Lai et al. 
1993). Members of this family of transcription factors share a highly conserved stretch of 100 amino acids containing a modified helix-turn-helix motif located in the middle of the protein. The similarity of these proteins is limited to the DNA-binding domain, suggesting that the amino- and carboxy-terminal domains have different functions in the various family members.

Mutations in the whn gene were found in three nude alleles, the mouse $n u$, the rat $r n u^{r}$, and the rat $r n u$ (Nehls et al. 1994; Segre et al. 1995). In the mouse nu allele, a single base-pair deletion in the whn coding sequence results in a frame shift and premature termination in the central putative DNA-binding domain. The rat $r n u^{r}$ allele also contains a mutation that disrupts the DNAbinding domain. The second rat allele, rnu, contains a nonsense mutation in the carboxy-terminal domain, which is proline-rich and possesses a high content of negatively charged amino acids characteristic of transcriptional transactivation domains. Thus, all of these mutations should result in nonfunctional Whn protcins.

Although whn was reported to be expressed in the skin and thymus, neither the specific cell type nor the consequence of lack of whn function was determined originally (Nehls et al. 1994; Segre et al. 1995). Recently, whn was shown to be expressed in the epithelial cells of the thymus, and its absence prevented normal thymic development through an as-yet-unidentified mechanism (Nehls et al. 1996). Here we show that Whn is expressed specifically in the epithelial cells of the skin, and that it plays a critical role in maintaining the balance between keratinocyte growth and differentiation. Alteration of this balance can explain the hair and skin phenotype of the nude mouse, and suggests that similar alterations could occur during the development of the thymic epithelium.

\section{Results}

Epithelial-specific expression of whn correlates with an intrinsic keratinocyte defect in nude mouse hair development

Although whn was shown to be expressed in the skin, the precise cell type was not determined. To address this question, we performed a Northern blot analysis of RNA derived from primary mouse and rat keratinocytes, mouse and rat dermal fibroblasts, and rat dermal papilla cells. A whn transcript of the expected size was expressed in keratinocytes and not in mesenchymal cells of either type (Fig. 1A). Our initial analysis was performed with unfractionated primary keratinocytes. Because lack of whn function affects hair follicle formation severely, we determined whether whn expression was confined to keratinocytes of the hair folliclc. Newborn mouse skin does not possess mature hair follicles, but instead contains follicle precursors called hair buds, which consist of specific keratinocyte aggregates. Hair buds can be separated from interfollicular keratinocytes by Ficoll gradient centrifugation (Weinberg et al. 1993). Northern blot analysis of RNA derived from both cultured hair buds

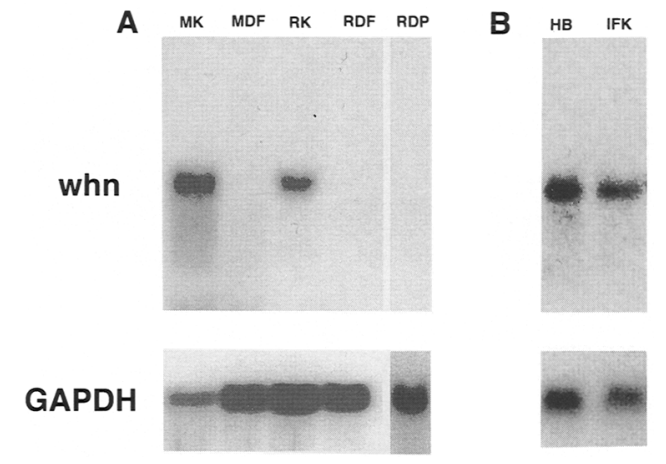

Figure 1. Specific pattern of whn expression in epithelial and mesenchymal skin cell populations. Northern blot analysis of RNA isolated from primary keratinocytes, primary dermal fibroblasts, or dermal papilla cells. The blot was first hybridized with a mouse whn cDNA probe, then with a GAPDH probe to assess RNA loading. The $4 \mathrm{~kb}$ whn transcript is indicated. (A) Primary mouse keratinocytes $(\mathrm{MK})$; primary mouse dermal fibroblasts $(\mathrm{MDF})$; primary rat keratinocytes $(\mathrm{RK})$; primary rat dermal fibroblasts (RDF); primary rat dermal papilla cells (RDP). $(B)$ Cultured mouse hair buds $(\mathrm{HB})$; cultured mouse interfollicular keratinocytes (IFK).

and interfollicular keratinocytes showed similar whn mRNA levels (Fig. 1B). Hence, whn expression is epithelial-cell-specific in skin, but is not confined to the keratinocyte progenitors of the hair follicle.

The keratinocyte-specific pattern of whn expression suggested that the functional defect in nude mouse hair development lies in the epithelial cells. We tested this possibility using a recently developed hair reconstitution grafting assay (Lichti et al. 1993; Weinberg et al. 1993). Hair buds were isolated from either wild-type Swiss ( wh $n^{+1+}$ ) or nude (Swiss whn ${ }^{-1-}$ ) newborn mouse pups and grafted with rat dermal papilla cells onto nude mice (Fig. 2). In contrast to the hair formed in control grafts (Fig. 2A,C,E), the hair formed by nude mouse hair buds was sparse and the follicles often lacked the inner root sheath, cuticle, and cortex (Fig. 2D,F). In addition, the hair follicles frequently formed cysts that were composed of bundles of keratinized material (Fig. 2D,F). This histological pattern is similar to that observed in the nude mouse (Köpf-Maier et al. 1990; Sundberg 1994). Thus, aberrant hair follicle formation in nude animals results from an intrinsic defect of keratinocytes, and correlates with the keratinocyte-specific pattern of whn expression.

Nude mouse keratinocytes display a normal mitogenic response under basal, growing conditions

The fact that whn is expressed equally in follicular and interfollicular keratinocytes (Fig. 1B) and that the keratinization of the epidermis of the nude mouse is also abnormal suggestcd that lack of whn function might alter the growth and differentiation properties of keratinocytes in general, rather than only those in the hair follicles. To test this hypothesis, primary keratinocytes 

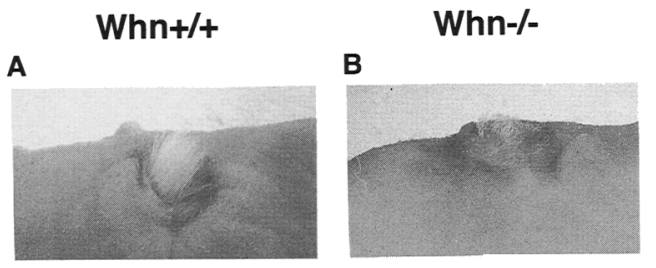

C

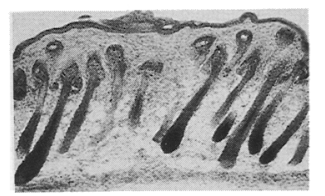

E

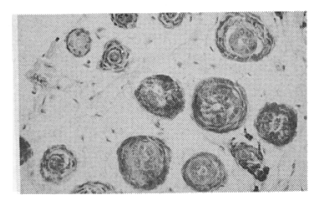

D

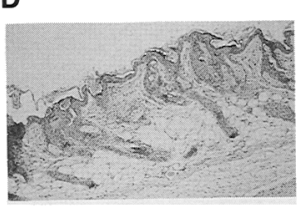

$\mathbf{F}$

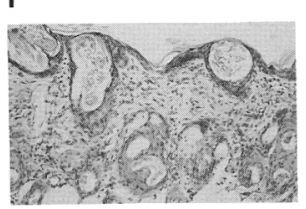

Figure 2. Defective hair reconstitution capability of nude mouse keratinocytes. Skin grafting hair reconstitution assays using hair buds derived from wild-type vs. nude mouse newborn skins were performed as described in Materials and Methods. Grafts from either wild-type $(A)$ or nude $(B)$ keratinocytes are shown. Hematoxylin- and eosin-stained longitudinal and cross sections are from wild-type $(C, E)$ and nude $(D, F)$ hair buds, respectively $(10 \times$ and $20 \times$, respectively).

derived from nude and wild-type mice were cultured under well-defined in vitro conditions. Primary keratinocytes maintained in medium at low calcium concentrations $(0.05 \mathrm{~mm})$ behave like cells of the basal layer of the epidermis (Hennings et al. 1980). The cultured cells proliferate continuously even after confluence is attained, constantly shedding new cells into the medium. To compare the mitogenic responses of wild-type and nude keratinocytes, cells of both types were growth-arrested by serum starvation for $36 \mathrm{hr}$. DNA synthesis was then measured in these cultures at fixed time intervals after serum and epidermal growth factor (EGF) stimulation (Fig. 3A). These experiments indicated that nude mouse keratinocytes entered $\mathrm{S}$ phase with the same kinetics as control cultures, and that there was no significant difference between the nude or wild-type keratinocytes in the magnitude of their mitogenic responses to serum and EGF. These findings were confirmed by determining the nuclear labeling index of control versus nude keratinocytes. A 3-hr bromodeoxyuridine (BrdU) pulse-labeling showed similar numbers of $\mathrm{whn}^{-1-}$ and whn ${ }^{+1+}$ cells in S phase (Fig. 3B). Thus, these data suggest that the mitogenic response of nude mouse keratinocytes under basal, proliferating conditions is intrinsically normal.

$\mathrm{whn}^{-1-}$ keratinocytes have an increased sensitivity to TPA, but not calcium- or TGF- $\beta$-induced growth arrest

Because the mitogenic response of $w^{-1-}$ keratinocytes appears normal, we examined whether they have an increased propensity to differentiate. In cultured keratinocytes, an increase in the extracellular calcium concentration triggers growth arrest and induces a program of terminal differentiation similar to that observed in the suprabasal layers of the epidermis (Hennings et al. 1980; Hennings and Holbrook 1983). Like calcium, treatment of cultured keratinocytes with the phorbol ester TPA (12-O-tetradecanoylphorbol-13-acetate) also induces growth arrest and several important aspects of keratinocyte differentiation (Dlugosz and Yuspa 1993; see below). In contrast, TGF- $\beta$ treatment induces only growth arrest and not markers of differentiation (Coffey et al. 1988; C. Missero and G.P. Dotto, unpubl.). We compared the sensitivity of $w h n^{-1-}$ and $w h n^{+1+}$ cells with the growth-inhibitory effects of these agents. As shown in Figure 4A, the ability of $w h n^{-1-}$ keratinocytes to be growth-arrested in response to calcium and TGF- $\beta 1$ did not differ significantly from wild-type cells. However, nude mouse keratinocytes were much more susceptible to the effects of TPA than control cells. To fur-

Figure 3. Normal mitogenic response and increased involucrin expression of wildtype vs. nude primary keratinocytes. $(A)$ Wild-type $\left(\mathrm{whn}^{+1+}\right.$ ) (solid bar) or nude (whn ${ }^{-1-}$ ) (open bar) keratinocytes were serum-starved for $36 \mathrm{hr}$ before addition of serum and EGF $(10 \mathrm{ng} / \mathrm{ml}) .\left[{ }^{3} \mathrm{H}\right]$-thymidine incorporation was measured in duplicate wells at the indicated times (hr) after release from growth arrest, and is expressed as percentage of the 30 -hr time point. $|B|$ Nuclear labeling index and number of involucrin positive cells were determined simultaneously in wild-type $\left(\mathrm{whn}^{+1+}\right)$ and nude ( $w^{-1-} n^{-1}$ keratinocytes as described in Materials and Methods. In each case 600-900 cells corresponding to at least 10 different fields were counted.

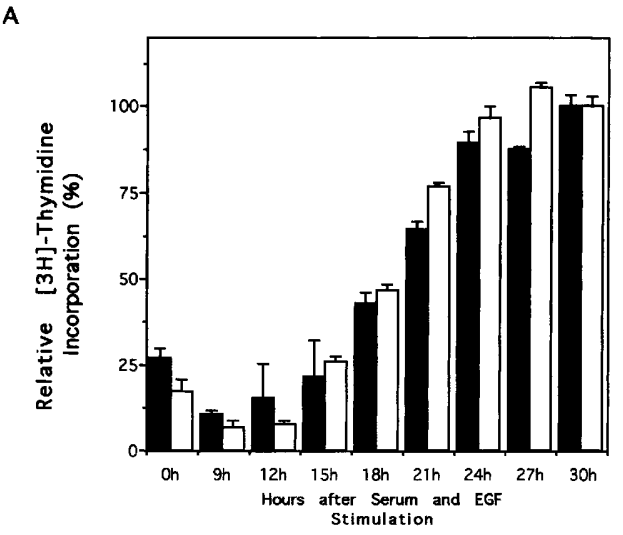

B

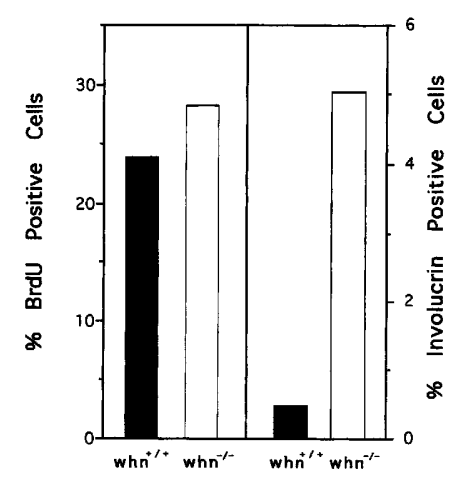


A

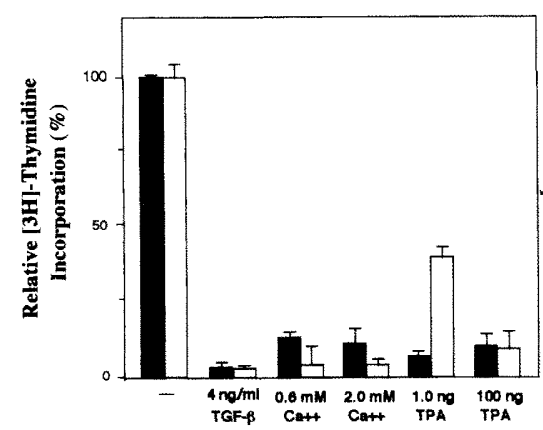

B

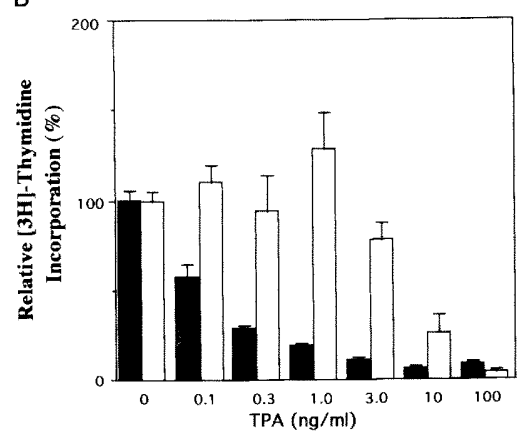

Figure 4. Increased sensitivity of nude mouse keratinocytes to TPA, but not calcium or TGF- $\beta$. $(A)$ Primary keratinocytes were either untreated or incubated for 24 hrs in the presence of calcium $10.6 \mathrm{~mm}$ or 2.0 $\mathrm{mM})$, TGF- $\beta 1$ ( $4 \mathrm{ng} / \mathrm{ml})$, or TPA ( 1 or 100 $\mathrm{ng} / \mathrm{ml}$ ). DNA synthesis was assayed in duplicate wells by a 3 -hr $\left[{ }^{3} \mathrm{H}\right]$-thymidine pulselabeling at the end of the incubation period, and is expressed as percentage of the untreated controls. (Solid bar) whn ${ }^{-1-}$; (open bar) whn $" /+$. Keratinocytes were incubated for $24 \mathrm{hr}$ in the absence or presence of TPA at the indicated concentrations, and DNA synthesis was measured as described in $A$. (Solid bar) whn ${ }^{-1}$; (open bar) $\mathrm{whn}^{+1+}$. ther substantiate the greater sensitivity of whn ${ }^{-/-}$cells to TPA, we tested the response to TPA at much lower concentrations. Very significant inhibitory effects were observed at concentrations as low as 0.1 or $0.3 \mathrm{ng} / \mathrm{ml}$, which is 100 -fold lower than the concentration necessary to affect control cells (Fig. 4B). This increased sensitivity was confirmed by determination of the nuclear labeling index by BrdU incorporation (data not shown). Thus, nude mouse keratinocytes are normal in their growth-inhibitory response to calcium and TGF- $\beta$, but have a much greater sensitivity to the effects of TPA.

\section{Nude mouse keratinocytes display an increased propensity to differentiate along the TPA signaling pathway}

As discussed, calcium triggers a terminal differentiation program in cultured keratinocytes that mimics that observed in the epidermis, including the induction of both early and late differentiation markers (Hennings et al. 1980|. TPA can also induce expression of some of these markers (involucrin, filaggrin, and loricrin), but fails to induce, or actually inhibits, others (keratins 1 and 10; Dlugosz and Yuspa 1993). We compared differentiation marker expression in $w h n^{-1-}$ versus $w h n^{+1+}$ keratinocytes under basal conditions and after induction of differentiation with either calcium or TPA.

Filaggrin, an intermediate filament-associated protein that functions to aggregrate keratins in the stratum corneum, is synthesized initially as a large molecular weight precursor, profilaggrin, in the granular layer (Resing et al. 1989). Western blot analysis showed that profilaggrin was expressed at very low levels in wild-type cultures under basal conditions and was induced in response to both calcium (Fig. 5) and TPA (Fig. 6). Interestingly, the level of profilaggrin was substantially higher in whn $n^{-1-}$ keratinocytes even under basal, proliferating conditions. These increased levels were similar to those observed in control cells after calcium and TPA treatment.

Involucrin, a component of the cornified envelope, is expressed at low levels in proliferating keratinocytes. Immunoblotting with anti-involucrin antibodies showed that involucrin expression, like filaggrin, was higher in nude mouse keratinocytes than in control cultures under both basal conditions and after exposure to stepwise increases in calcium concentrations $(F i g .5 \mathrm{~B} \mid$. These findings were confirmed by immunofluorescence experiments that showed 10 -fold more involucrin-positive cells in nude primary cultures than in control cultures even under basal, growing conditions (Fig. 3B). A similar fraction of involucrin-positive cells $(10 \%)$ still synthesizing DNA (as assessed by bromodeoxyuridine labeling)

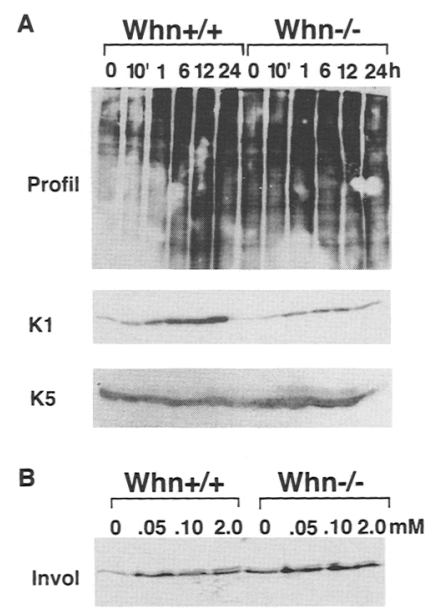

Figure 5. Aberrant differentiation marker expression in nude mouse keratinocytes under basal conditions and after calcium-induced differentiation. (A) Control $\left(\mathrm{whn}^{+1+}\right)$ or nude | whn ${ }^{-1-} \mid$ primary cultures were grown in low calcium medium and induced to differentiated by addition of $2.0 \mathrm{~mm}$ calcium for the indicated times. Total cell extracts were normalized for protein content, electrophoresed on a $7.5 \%$ SDS-PAGE, and immunoblotted with affinity purified antisera against profilaggrin (Profil), keratin 1 (K1), and keratin 5 (K5). Filaggrin is synthesized as a high molecular weight precursor, profilaggrin, which is subsequently processed. The diffuse bands correspond to the multiple products of this processing. (B) Nude (whn ${ }^{-1}$ ) or wild-type ( $w n^{+/+}$) keratinocytes exposed to increasing calcium concentrations $(\mathrm{mM})$ for $24 \mathrm{hr}$ were analyzed for involucrin (Invol) expression by immunoblotting with specific antibodies. 


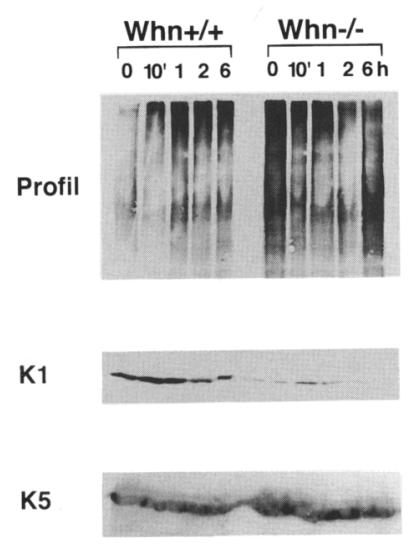

Figure 6. Abnormal differentiation marker profile in control and nude mouse keratinocytes under proliferating conditions and after TPA exposure. Cell extracts were prepared from wildtype $\left(\mathrm{whn}^{+1+}\right)$ or nude $\left(\mathrm{whn}^{-1-}\right)$ keratinocytes after treatment with $10 \mathrm{ng} / \mathrm{ml}$ TPA for the indicated times (hr). Samples were normalized for protein concentration, separated on a $7.5 \%$ SDS PAGE, and immunoblotted with antiprofilaggrin (Profil), antikeratin $1(\mathrm{~K} 1)$, and antikeratin 5 (K5) antibodies. The lesser amounts of profilaggrin detected at later times in both control and nude mouse keratinocytes is likely attributable to its conversion to the mature filaggrin products.

was found in both wild-type and nude keratinocyte cultures.

Keratin $l(K 1)$ is a marker of the spinous epidermal layer, and was induced in wild-type primary keratinocytes after $1 \mathrm{hr}$ of calcium treatment (Fig. 5). As reported previously (Dlugosz and Yuspa 1993), exposure of wild-type cells to TPA inhibited K1 expression by $6 \mathrm{hr}$ (Fig. 6). In nude mouse keratinocytes, Kl expression levels were significantly lower than in control cells under both basal conditions and after exposure to calcium (Fig. 5). TPA treatment of nude mouse keratinocytes reduced further the amount of the Kl protein expression (Fig. 6). Keratin $5(\mathrm{~K} 5)$ is a marker of the basal epidermal layer, and its expression does not change in cultured keratinocytes after treatment with either calcium or TPA. Unlike the previous markers, immunoblotting experiments indicated that $\mathrm{K} 5$ was expressed equally in $\mathrm{whn}^{+/+}$and whn ${ }^{-1-}$ keratinocytes.

Thus, the increased expression of filaggrin and involucrin and the reduced expression of keratin 1 in keratinocytes with a mutated whn gene resembles that of TPA-treated, wild-type cells. These data show that nude cells possess a greater propensity to express markers of differentiation under basal, proliferating conditions, and suggest that in nude keratinocytes the differentiation/ TPA-mediated signaling pathway may be specifically activated.

Whn expression can specifically suppress the promoter activity of differentiation/TPA-responsive genes

Because sequence analysis indicates that Whn is a putative transcription factor, we tested directly whether its expression can affect the promoter activity of differentiation-inducible genes. The full-length mouse whn cDNA was placed under the control of the CMV promoter in a mammalian expression vector. Whn function was then assessed by cotransfecting this plasmid with constructs in which various promoters were fused to the luciferase reporter gene.

The activity of the involucrin promoter, in parallel with the induction of its corresponding gene product, is induced in primary keratinocytes in response to both calcium (data not shown) and TPA (Fig. 7A, right). Consistent with the observation that involucrin protein levels are higher in nude mouse keratinocytes, transient transfection assays under basal conditions showed a higher involucrin promoter activity in $\mathrm{whn}^{-1-}$ cells relative to $\mathrm{whn}^{+1+}$ keratinocytes (Fig. 7A, left). The activity of the involucrin promoter in both types of cells was then examined following expression of the Whn factor itself. We found that involucrin promoter activity was suppressed dramatically by Whn in a dose-dependent

A
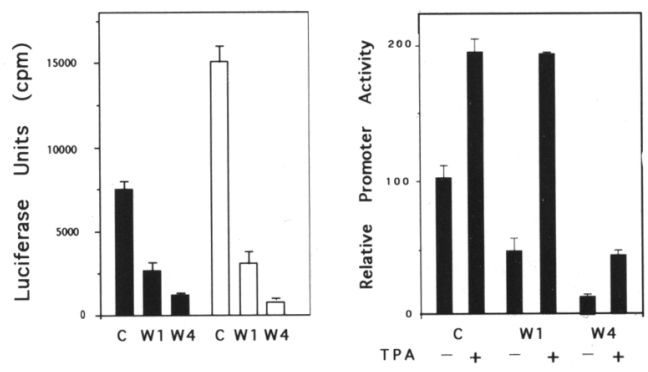

B

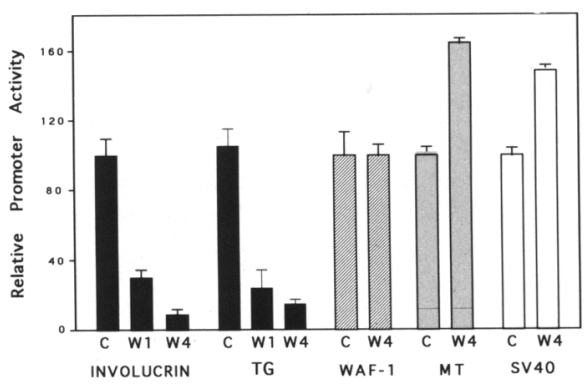

Figure 7. Specific inhibitory effects of Whn on differentiation/ TPA-responsive promoters. $(A)$ (Left) Wild-type or nude keratinocytes were cotransfected with either an involucrin promoterluciferase construct together with either 1 or $4 \mu \mathrm{g}$ of a whn expression plasmid (W1, W4), or $4 \mu \mathrm{g}$ of an empty vector control (C). (Solid bar) whn ${ }^{+1+}$; (open bar) whn ${ }^{-1}$. (Right) Wild-type keratinocytes transfected with the same constructs as in the left panel were either untreated $\mid-$ ) or treated with $100 \mathrm{ng} / \mathrm{ml}$ TPA $(+\mid$ for $8 \mathrm{hr}$ before harvesting. (B) Wild-type keratinocytes were cotransfected with various promoter-luciferase reporter gene constructs and either 1 or $4 \mu \mathrm{g}$ of the whn expression plasmid (W1, W4) or $4 \mu \mathrm{g}$ of the vector control (C). The promoters tested included involucrin, transglutaminase (TG), WAF-1, metallothionein $(\mathrm{MT})$, or SV40, and were as described in Materials and Methods. 
manner, and that Whn expression was much more in-

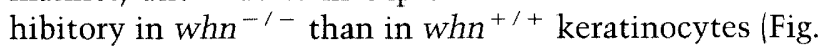
7A, left).

As discussed previously, involucrin promoter activity is induced in keratinocytes by TPA, so we examined whether whn expression could suppress this response. We found that whereas Whn was able to inhibit involucrin promoter activity under basal proliferating conditions, Whn did not block the TPA inducibility of this promoter (Fig. 7A, right).

Epidermal transglutaminase is also a marker of differentiation and is responsible for cross-linking the components of the cornified envelope. Like involucrin, transglutaminase expression is induced by both calcium and TPA in culture (Filvaroff et al. 1995). The Whn expression construct was cotransfected with a reporter plasmid containing the transglutaminase promoter (Mariniello et al. 1995|. Similar to the results obtained with the involucrin promoter, Whn inhibited transglutaminase promoter activity in a dose-dependent manner (Fig. 7B).

The expression of the cell-cycle inhibitor Waf- 1 (p21, Cip 1; El-Deiry et al. 1993; Harper et al. 1993; Xiong et al. 1993 ) is increased at the transcriptional level in keratinocytes induced to differentiate by calcium, but not TPA (Missero et al. 1995; Missero and Dotto 1996). Unlike its inhibition of the involucrin and transglutaminase promoters, Whn expression had no effect on the activity of WAF-1 promoter in cotransfection assays (Fig. 7B).

To further test the specificity of Whn, the whn expression plasmid was cotransfected with the luciferase reporter gene fused to either a minimal metallothionein or SV40 promoter. Whn expression had no inhibitory effect and instead slightly stimulated both the minimal metallothionein and SV40 promoters (Fig. 7B).

Thus, expression of the Whn factor specifically repressed the promoters of two differentiation/TPA-responsive genes (involucrin and transglutaminase), but had little or no effect on the promoter of a differentiation/calcium-responsive gene (Waf-1) or other unrelated promoters.

\section{Is Whn a transcriptional activator or repressor?}

To map the region of Whn responsible for repression of the involucrin promoter, we constructed three Whn mutant proteins that contained either the amino-terminal plus the DNA-binding domains, only the DNA-binding domain, or the DNA-binding domain plus the carboxyterminal regions (Fig. 8A). The ability of these mutant proteins to inhibit the involucrin promoter was tested in transient transfection assays. The Whn mutant that contains the DNA-binding domain plus the carboxyl terminus was as effective as the full-length, wild-type protein in inhibiting the involucrin promoter. In contrast, the other two mutants that possess only the DNA-binding or the amino-terminus plus DNA-binding domains were relatively ineffective.

To determine whether Whn directly functions as a repressor, the DNA-binding domain of the yeast Gal4 protein was fused to either full-length Whn, or its separate
A

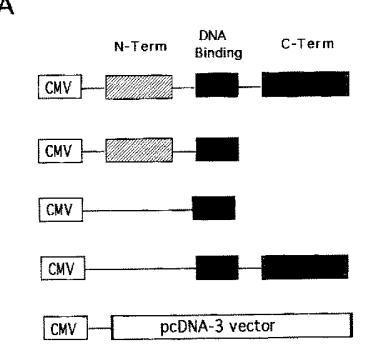

B
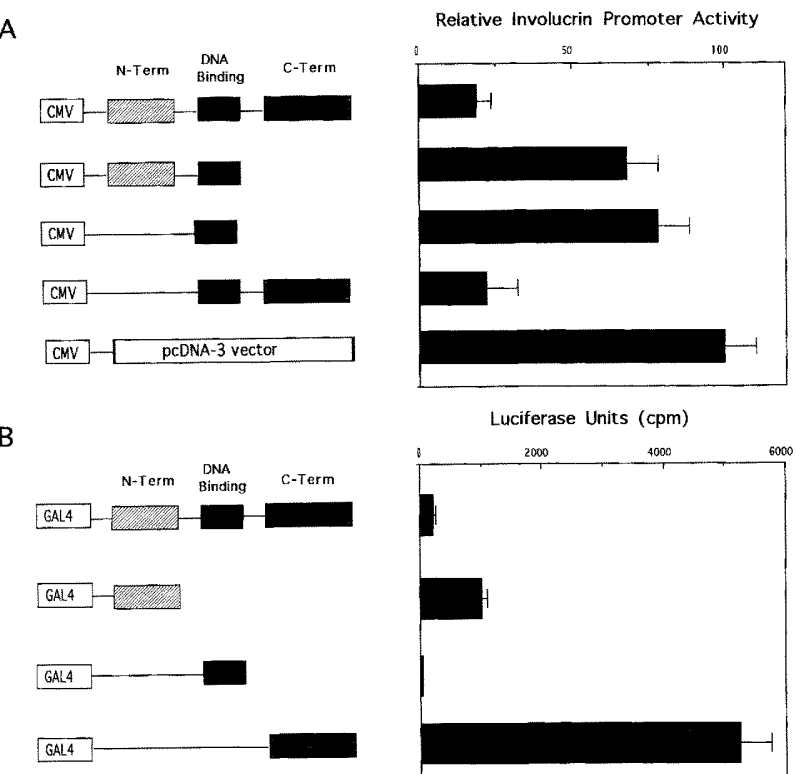

Luciferase Units (cpm)

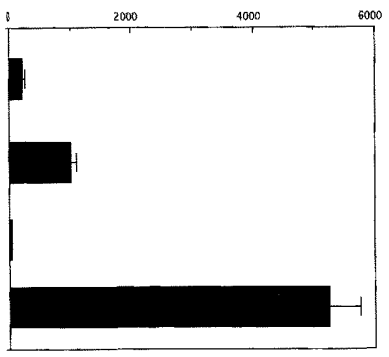

Figure 8. Functional mapping of the involucrin inhibitory re gion of Whn to its carboxy-terminal, transactivation domain. (A) Either the full-length whn cDNA, or truncated regions containing the indicated domains were placed under control of the CMV promoter in the pcDNA-3 mammalian expression vector. These constructs were cotransfected with the involucrin promoter-luciferase reporter plasmids into wild-type keratinocytes. Luciferase activity was measured 3 days after transfection, and is expressed relative to involucrin promoter activity in cells transfected with the vector control. $(B)$ Either the fulllength whn cDNA or truncated regions containing the indicated domains were fused to the Gal4 DNA-binding domain in the pSG424 mammalian expression vector. These plasmids were cotransfected with the yeast UAS (upstream activating sequencel promoter fused to a luciferase reporter gene. Transiently transfected cells were harvested after 3 days and luciferase activity was determined as described in Materials and Methods.

amino-terminal, DNA-binding, or carboxy-terminal regions (Fig. 8B). Transient transfection assays were then performed with plasmids expressing these fusion proteins, and a reporter plasmid containing the luciferase gene under the control of a promoter with a Gal4 binding site. Only the carboxy-terminal Whn fusion protein was capable of stimulating transcription (Fig. 8B). Neither the full-length protein nor its amino terminal or putative DNA-binding regions increased promoter activity. Consistent with these results, the carboxy-terminal domain is the only region that bears resemblance to an activation domain of a transcription factor.

Thus, the carboxy-terminal domain of Whn is essential for inhibition of the involucrin promoter, and this domain appears to function as a transcriptional activator.

\section{Discussion}

Whn, the gene responsible for the nude mouse phenotype (Nehls et al. 1996), is expressed specifically in ke- 
ratinocytes and not dermal papilla or interfollicular fibroblasts. Using a hair reconstitution grafting assay, we have demonstrated that the abnormal hair development found in nude mice is caused by an intrinsic epithelial cell defect. Finally, Whn functions to control the balance between keratinocyte growth and differentiation, and interferes at the transcriptional level with a selected number of differentiation-responsive genes.

whn $n^{-1-}$ keratinocytes examined under well-defined in vitro conditions exhibited significantly altered differentiation behavior, consistent with the abnormal keratinization process observed in both nude mouse skin (Köpf-Maier et al. 1990; Sundberg 1994) and hair follicles reconstituted with whn $^{-1-}$ keratinocytes. Two late markers of keratinocyte differentiation, involucrin and filaggrin, were expressed at much higher levels in whn ${ }^{-1}$ cells than in wild-type keratinocytes even under basal, proliferating conditions. In contrast, keratin 1 , an early differentiation marker, was present at much lower amounts in nude mouse keratinocytes under all conditions tested.

The altered pattern of differentiation markers observed in nude mouse keratinocytes under basal conditions is similar to that observed in wild-type cells after exposure to TPA (Dlugosz and Yuspa 1993). In parallel with these findings, nude mouse keratinocytes exhibited a markedly increased sensitivity to TPA, but not to other modulators of growth and differentiation such as calcium and TGF- $\beta$. These results suggest that the TPA signaling pathway is activated in nude mouse keratinocytes even under basal, proliferating conditions. There might be a similar increase in the sensitivity of nude mouse skin to TPA in vivo; however, a direct test of this possibility is hampered by the thick stratum corneum characteristic of these animals that interferes with TPA penetration.

The lack of whn function could cause a constitutive increase of the early, immediate events triggered by TPA, such as the activation of protein kinase $\mathrm{C}$ ( $\mathrm{PKC}$; Kikkawa et al. 1989/ and the AP-1 transcription factor (Angel and Karin 1991). Involucrin, as well as other keratinocyte differentiation markers, contain AP-1 consensus sequences in their promoters, and AP-1 family members have been postulated to play an important role in the induction of these genes during differentiation (Rothnagel et al. 1993; Saunders et al. 1993; Takahashi and Iizuka 1993; Gandarillas and Watt 1995, and references therein; Welter and Eckert 1995). Involucrin promoter activity is increased in whn ${ }^{-1-}$ keratinocytes, and overexpression of whn inhibits the activity of this promoter. However, whn expression did not suppress the TPA inducibility of the involucrin promoter and, in fact, increased the relative induction of this promoter by TPA. Similarly, whn had no effect on the ability of TPA to induce the promoter activity of c-fos (data not shown), a well-established TPA-responsive gene and component of the AP-1 transcription complex (Angel and Karin 1991). Thus, Whn function is unlikely to affect the PKC pathway directly responsible for AP-1 activation. However, several PKC isozymes exist that contribute to the or- dered program of keratinocyte differentiation (Denning et al. 1995), and it is possible that Whn specifically controls one or more of these isoforms in a manner that is not related to AP-1 induction.

Whn's inhibitory effects were specific for promoters of differentiation markers, such as involucrin and transglutaminase, which are induced by both TPA and calcium. In contrast, Whn had no effect on the promotor of another differentiation-responsive gene, Waf- 1 , which is induced specifically in response to calcium, but not TPA (Missero et al. 1995; Missero and Dotto 1996). The basal activities of both the minimal SV40 and metallothionein promoters were similarly unaffected.

One possible explanation for these results is that Whn acts as a direct repressor of the involucrin and transglutaminase promoters. Indeed, another winged-helix family member, v-Qin, has been shown to function as a direct transcriptional repressor when fused to a yeast Gal4 DNA-binding domain ( $\mathrm{Li}$ et al. 1995). The region of $\mathrm{v}$-Qin responsible for this direct repression was mapped to the carboxy-terminal domain of the protein. We have shown that the carboxy-terminal domain of Whn is also essential for the suppression of the involucrin promoter. However, when this region of Whn was fused to the Gal4 DNA-binding domain, it functioned as a transcriptional activator, rather than a repressor. This indicates that Whn functions primarily as a transcriptional activator, and that its inhibitory effects on the involucrin promoter are most likely indirect. Consistent with this intrepretation, the carboxy-terminus of Whn resembles the activation domains of other transcription factors in that it is proline-rich and contains a high content of negatively charged amino acids. Interestingly, the fusion of the fulllength Whn protein to the Gal4 DNA-binding domain was inactive, suggesting that the amino terminus of Whn could possess a negative regulatory function. Thus, Whn might act analogously to another winged-helix protein, Ets-1, which undergoes an activating conformational change upon binding to its specific DNA recognition sequence (Peterson et al. 1995).

In vivo treatment of mouse skin with TPA induces keratinocyte stem cells to divide and produce an increased number of transiently amplifying and more committed populations (Cotsarelis et al. 1990). Given the fact that Whn-deficient keratinocytes are already in a TPA-primed state, we suggest that the number of keratinocyte stem cells and their differentiated progeny is altered similarly in nude mice. Consistent with an enhanced production of differentiated cells, the skin of the nude animals shows an increased number of cornified layers and hair follicle cysts that can account for the apparent lack of hair. In addition to the skin phenotype, nude mice have a total loss of thymus function, which is attributable to a defect in the ectodermal component of this organ during development (Nehls et al. 1996). The thymus and the skin epithelia are remarkably similar, and share the expression of a number of genes including specific keratins (Farr and Braddy 1989; Barrett et al. 1992). These two tissues may also share similar regulatory networks that determine the balance between 
growth and differentiation. Some components of this network may be under the direct transcriptional control of whn. Identification of these molecules will aid significantly in the elucidation of the mechanisms underlying self-renewal of these epithelia.

\section{Materials and methods}

Cells

Primary keratinocytes were prepared from either newborn nude (Swiss ${ }^{n u / m u}$ ) or Swiss (Swiss ${ }^{+/+}$) mice and grown minimal essential medium at low calcium concentrations $(0.05 \mathrm{~mm})$ supplemented with $4 \%$ Chelex-treated fetal calf serum and epidermal growth factor (10 $\mathrm{ng} / \mathrm{ml}$; Collaborative Research) as described previously (Hennings et al. 1980). EGF was present in all experiments unless otherwise stated. Differentiation was induced by addition of $\mathrm{CaCl}_{2}$ (final concentration of $2 \mathrm{mM}$ ) or TPA ( 10 or $100 \mathrm{ng} / \mathrm{ml}$ in DMSO). TGF- $\beta$ was used at $4 \mathrm{ng} / \mathrm{ml}$. Hair buds and interfollicular keratinocytes were isolated from 1- to 2-day-old newborn mouse skin and purified by two sequential Ficoll gradient centifugation steps (Weinberg et al. 1993). These keratinocyte preparations were either used immediately for grafting experiments or cultured for 1 weck in low calcium medium.

Primary dermal papilla cells were dissected from 4- to 6-week-old Fisher rat vibrissal follicles as described by Jahoda and Oliver (1981). Papilla cells were passaged three times before grafting, the first two passages in Chang's medium (Irvine Scientific) and the last passage in DMEM supplemented with $10 \%$ fetal calf serum.

\section{Hair reconstitution grafting assay}

The hair reconstitution assays were performed using freshly prepared hair buds ( 2.5 skin equivalents/graft), rat dermal papilla cells $\left(8 \times 10^{6} / \mathrm{graft}\right)$, and Swiss $3 \mathrm{~T} 3 \mathrm{~J} 2 \mathrm{~J}$ fibroblasts $\left(2 \times 10^{6} /\right.$ graft; kindly provided by Dr. H. Green, Harvard Medical School, Boston, MA) as described previously (Lichti et al. 1993; Weinberg et al. 1993!. Prior to grafting, hair buds were combined with dermal papilla cells and Swiss $3 \mathrm{~T} 3$ fibroblasts, and subjected to centrifugation $(1000 \mathrm{~g}, 5 \mathrm{~min})$. These dense cell suspensions were then injected into silicon transplantation chambers implanted onto the backs of nude mice. Transplantation chambers were removed 1 week after grafting, and animals were sacrificed 6 weeks later. Grafts were fixed in 10\% formalin (Fisher Scientific), then embedded in paraffin. Sections were examined after hematoxylin and eosin staining.

\section{Whn and fusion protein constructs}

The full-length mouse whn cDNA was cloned using RT-PCR with oligonucleotides corresponding to nucleotides (nt) 53-73 and the reverse complement nt 2115-2135 of the mouse cDNA sequence (Nehls et al. 1994). The nucleotide sequence was confirmed by sequencing. The mouse whn cDNA was then inserted into the pcDNA-3 mammalian expression vector /Invitrogen). The following Whn mutant proteins were constructed by PCR: (1) amino-terminal region plus the DNA-binding domain (nt 94-1230); (2) DNA-binding domain only (nt 7931230); and (3) DNA-binding domain plus the carboxy-terminal region (nt 793-2040).

The Gal4-Whn fusion proteins were constructed using mouse whn nucleotide sequences corresponding to the fulllength (nt 97-2060), amino-terminal (nt 97-792), DNA-binding (nt 774-1239), or carboxy-terminal (nt 1202-2060) domains of the protein. These nucleotide sequences were inserted in frame with the Gal4 DNA-binding domain in the pSG424 vector /Sadowski and Ptashne 1989).

\section{Northern blot analysis}

Total RNA was isolated from keratinocytes, dermal fibroblasts, and dermal papilla cells as described /Chomczynski and Sacchi 1987 ), separated on $1.2 \%$ agarose/formaldehyde gels, and transferred to Hybond $\mathrm{N}$ membranes (Amersham). Blots were probed with a ${ }^{32}$ p-labeled whn probe corresponding to nt 677-1042 of the mouse cDNA sequence (Nehls et al. 1994).

\section{Immunoblotting and antibodies}

Proteins, extracted from keratinocytes by addition of boiling sample buffer, were normalized for protein concentration (Bradford assay; Bio-Rad Laboratories), separated on SDS/7.5\% polyacrylamide gels, and transferred to Immobilon-P membranes (Millipore). Immunoblots were probed with affinity-purified polyclonal antibodies against filaggrin, keratin 1, keratin 5 /all kindly provided by Dr. S.H. Yuspa, National Cancer Institute, Bethesda, MD), and involucrin (Berkeley Antibodies Company). Blots were developed using horseradish peroxidase-conjugated secondary antibodies and the ECL detection system /Amersham).

Transient transfections, luciferase assays, and reporter plasmids

Primary keratinocytes were transfected with plasmid DNAs using the DEAE-dextran technique (Selden 1992) in serum-free, low calcium medium for $2 \mathrm{hr}$ followed by a 1 min dimethylsulfoxide shock as described previously (Missero et al. 1995). Transfected cells were maintained in low calcium medium or treated with TPA for the last $8 \mathrm{hr}$ of the 72 -hr incubation period. In all instances, cells were harvested $72 \mathrm{hr}$ after transfection and luciferase activity was determined as described previously (Brasier et al. 1989). A plasmid encoding human growth hormone (pXGH5; Nichols Diagnostics Institute) was cotransfected $(1 \mu \mathrm{g} / 60 \mathrm{~mm}$ dish) to normalize luciferase values using the HGH-TGES kit (Nichols Diagnostics Institute) according to the manufacturer's instructions. Values are expressed as luciferase units $(\mathrm{cpm})$ per ng growth hormone.

The following promoters were used in promoter-luciferase fusion plasmids: (1) the human involucrin promoter (Carroll et al. 1993); (2) the human transglutaminase promoter obtained by PCR from the published sequence (Mariniello et al. 1995); (3) the human WAF-1 promoter (El-Deiry et al. 1993); (4) the minimal mouse metallothionein promoter (Pharmacia); (5) the miminal SV40 promoter (Promega); and $|6|$ the Gal4 binding sequence (Ornitz et al. 1991). The amount of promoter-reporter plasmid used $2 \mu \mathrm{g}$ per $60 \mathrm{~mm}$ dish.

\section{Bromodeoxyuridine labeling and involucrin immunofluorescence}

Primary keratinocytes were incubated in the presence of bromodeoxyuridine (BrdU) (Amersham) for $3 \mathrm{hr}$. Cultures were fixed with $2 \%$ paraformaldehyde for $15 \mathrm{~min}$ at room temperature, permeabilized with $0.1 \%$ Tween 20 in PBS, and incubated with mouse anti-BrdU (Becton Dickinson) and rabbit anti-involucrin antibodies (Berkeley Antibodies Company) for $1 \mathrm{hr}$. Samples were then washed with PBS and incubated for $1 \mathrm{hr}$ with antimouse rhodamine conjugated IgG (Cappel) and biotinylated goat antirabbit IgG (Vector). After washing, fluorescein-conju- 
gated streptavidin (Amersham) was added for $30 \mathrm{~min}$, samples were extensively washed, and mounted in $0.1 \mathrm{M} \mathrm{n}$-propylgallate in glycerol.

\section{Acknowledgments}

We are grateful to Ed Rose for maintaining the nude mouse colony and Rob Little for excellent technical assistance. We also thank Drs. Lorin Weiner, Caterina Missero, David Prowse, and Enzo Calautti for critical reading of the manuscript. This work was supported by National Institutes of Health Grants AR39190 and CAl6038 to G.P.D.

The publication costs of this article were defrayed in part by payment of page charges. This article must therefore be hereby marked "advertisement" in accordance with 18 USC section 1734 solely to indicate this fact.

\section{References}

Angel, P. and M. Karin. 1991. The role of Jun, Fos and the AP-1 complex in cell-proliferation and transformation. Biochim. Biophys. Acta. 1072: 129-157.

Barrett, T., M. Delvy, D. Kennedy, L. Lefrancois, L. Matis, A. Dent, S. Hedrick, and J. Bluestone. 1992. Mechanism of selftolerance of $\gamma \delta \mathrm{T}$ cells in epithelial tissue. I. Exp. Med. 175: 65-70.

Brasier, A., J. Tate, and J. Habener. 1989. Optimized use of the Firefly luciferase assay as a reporter gene in mammalian cell lines. BioTechniques 7: 1116-1121.

Calautti, E., C. Missero, P. Stein, R. Ezzell, and G.P. Dotto. 1995. fyn tyrosine kinase is involved in keratinocyte differentiation control. Genes \& Dev. 9: 2279-2291.

Carroll, J., K. Albers, J. Garlick, R. Harrington, and L. Taichman. 1993. Tissue- and stratum-specific expression of the human involucrin promoter in transgenic mice. Proc. Natl. Acad. Sci. 90: 10270-10274.

Chomezynski, P. and N. Sacchi. 1987. Single-step method of RNA isolation by acid guanidinium thiocyanate-phenolchloroform extraction. Anal. Biochem. 162: 156-159.

Coffey, R.J., Jr., N.J. Snipes, C.C. Bascom, R. Graves-Deal, Y. Pennington, B.E. Weissman, and H.L. Moses. 1988. Growth modulation of mouse keratinocytes by transforming growth factors. Cancer Res. 48: 1596-1602.

Cotsarelis, G., T.-T. Sun, and R. Lavker. 1990. Label-retaining cells reside in the bulge area of pilosebaceous unit: Implications for follicular stem cells, hair cycle, and skin carcinogenesis. Cell 61: 1329-1337.

Denning, M., A.A. Dlugosz, E.K. Williams, Z. Szallasi, P. Blumberg, and S.H. Yuspa. 1995. Specific protein kinase C isozymes mediate the induction of keratinocyte differentiation markers by calcium. Cell Growth Differ. 6: 149-157.

Dlugosz, A. and S.H. Yuspa. 1993. Coordinate changes in gene expression which mark the spinous to granular cell transition in epidermis are regulated by protein kinase C. $J$. Cell Biol. 120: 217-225.

El-Deiry, W., T. Tokino, V. Velculescu, D. Levy, R. Parsons, J. Trent, D. Lin, W. Mercer, K. Kinzler, and B. Vogelstein. 1993. $W A F 1$, a potential mediator of p53 tumor suppression. Cell 75: $817-825$.

Farr, A. and S. Braddy. 1989. Patterns of keratin expression in the murine thymus. Anat. Rec. 224: 374-378.

Filvaroff, E., D. Stern, and G.P. Dotto. 1990. Tyrosine phosphorylation is an early and specific event involved in primary keratinocyte differentiation. Mol. Cell. Biol. 10: 1164-1173.

Flanagan, S. 1966. "Nude," a new hairless gene with pleiotropic effects in the mouse. Genet. Res. 8: 295-309.

Gandarillas, A. and F. Watt. 1995. Changes in expression of members of the fos and jun families and myc network during terminal differentiation of human keratinocytes. Oncogene 11: 1403-1407.

Hardy, M.H. 1992. The secret life of the hair follicle. Trends Genet. 8: 55-61.

Harper, J., G. Adami, N. Wei, K. Keyomarsi, and S. Elledge. 1993. The p $21 \mathrm{Cdk}$-interacting protein Cipl is a potent inhibitor of Gl cyclin-dependent kinases. Cell 75: 805-816.

Hennings, H. and K. Holbrook. 1983. Calcium regulation of cell-cell contact and differentiation of epidermal cells in culture: An ultrastructural study. Exp. Cell Res. 143: 127-142.

Hennings, H., D. Michael, C. Cheng, P. Steinert, K. Holbrook, and S.H. Yuspa. 1980. Calcium regulation of growth and differentiation of mouse epidermal cells in culture. Cell 19: 245-254.

Jahoda, C. and R. Oliver. 1981. The growth of vibrissa dermal papilla cells in vitro. Br. J. Dermatol. 105: 623-627.

Kikkawa, U., A. Kishimoto, and Y. Nishizuka. 1989. The protein kinase $\mathrm{C}$ family: Heterogeneity and its implications. Annu. Rev. Biochem. 58: 31-44.

Köpf-Maier, V. Mboneko, and H. Merker. 1990. Nude mice are not hairless. Acta. Anat. 139: 178-190.

Lai, E., K. Clark, S. Burley, and J. Darnell. 1993. Hepatocyte nuclear factor $3 /$ fork head or "winged helix" proteins: A family of transcription factors of diverse biologic function. Proc. Natl. Acad. Sci. 90: 10421-10423.

Li, J., H. Chang, E. Lai, E. Parker, and P. Vogt. 1995. The Oncogene qin codes for a transcriptional repressor. Cancer Res. 55: $5540-5544$.

Lichti, U., W. Weinberg, L. Goodman, S. Ledbetter, T. Dooley, D. Morgan, and S.H. Yuspa. 1993. In vivo regulation of murine hair growth: Insights from grafting defined cell populations onto nude mice. I. Invest. Dermatol. 101: 124S-129S.

Mariniello, L., Q. Qin, B. Jessen, and R. Rice. 1995. Keratinocyte transglutaminase promoter analysis. Identification of functional response element. /. Biol. Chem. 270: 31358-31363.

Missero, C. and G.P. Dotto. 1996. p21 $21^{\text {WAF1/Cipl }}$ and terminal differentiation control of normal epithelial. Mol. Cell. Differ. 4: 1-16.

Missero, C., E. Calautti, R. Eckner, J. Chin, L.H. Tsai, D.M. Livingston, and G.P. Dotto. 1995. Involvement of the cellcycle inhibitor Cipl/WAF1 and the E1A-associated p300 protein in terminal differentiation. Proc. Natl. Acad. Sci. 92: 5451-5455.

Moscat, J., T. Fleming, C. Molloy, M. Lopez-Barahona, and S. Aaronson. 1989. The calcium signal for Balb/MK keratinocyte terminal differentiation induces sustained alterations in phosphoinositide metabolism without detectable protein kinase Cactivation. J. Biol. Chem. 264: 11228-11235.

Nehls, M., D. Pfeifer, M. Schorpp, H. Hedrich, and T. Boehm. 1994. New member of the winged-helix protein family disrupted in mouse and rat nude mutations. Nature 372: 103107.

Nehls, M., B. Kyewski, M. Messerle, R. Waldschutz, K. Schuddekopf, A. Smith, and T. Boehm. 1996. Two genetically separable steps in the differentiation of thymic epithelium. Science 272: 886-889.

Osada, S.-I., Y. Hashimoto, S. Nomura, Y. Kohno, K. Chida, O. Tajima, K. Kubo, K. Akimoto, H. Koizumi, Y. Kitamura, K. Suzuki, S. Ohno, and T. Kuroki. 1993. Predominant expression of $\mathrm{nPKCh} \eta$, a $\mathrm{Ca}^{2+}$-independent isoform of protein $\mathrm{ki}$ nase $C$ in epithelial tissues, in association with epithelial differentiation. Cell Growth Different. 4: 167-175.

Ornitz, D.M., W. Moreadith, and P. Leder. 1991. Binary system 
for regulating expression in mice: Targeting int-2 gene expression with yeast GAL4/UAS control elements. Proc. Natl. Acad. Sci. 88: 698-702.

Pantelouris, E. 1973. Athymic development in the mouse. Differentiation 1: 437-450.

Petersen, J., J. Skalicky, L. Donaldson, L. McIntosh, T. Alber, and B. Graves. 1995. Modulation of transcription factor Ets-1 DNA binding: DNA-induced unfolding of an $\alpha$ helix. Science 269: 1866-1868.

Resing, K.A., K.A. Walsh, J. Haugen-Scofield, and B.A. Dale. 1989. Identification of proteolytic cleavage sites in the conversion of profilaggrin to filaggrin in mammalian epidermis. J. Biol. Chem. 264: 1837-1845.

Rothnagel, J.A., D.A. Greenhalgh, T.A. Gagne, M.A. Longley, and D.R. Roop. 1993. Identification of a calcium-inducible epidermal-specific regulatory element in the 3 '-flanking region of the human keratin 1 gene. /. Invest. Dermatol. 101: $506-513$.

Sadowski, I. and M. Plashne. 1989. A vector for expressing GAL4(1-147) fusions in mammalian cells. Nucleic Acids Res. 17: 7539 .

Saunders, N., S. Bernacki, T. Vollberg, and A. Jetten. 1993. Regulation of transglutaminase type I expression in squamous differentiating rabbit tracheal epithelial cells and human epidermal keratinocytes: Effects of retinoic acid and phorbol esters. Mol. Endocrinol. 7: 387--398.

Segre, J., J. Nemhauser, B. Taylor, J. Nadeau, and E. Lander. 1995. Positional cloning of the nude Locus: Genctic, physi$\mathrm{cal}$, and transcription maps of the region and mutations in the mouse and rat. Genomics 28: 549-559.

Selden, R. 1992. Transfection using DEAE-dextran. In Current protocols in molecular biology (ed. F. Ausubel, R. Brent, R. Kingston, D. Moore, J. Seidman, J. Smith, and K. Struhl), pp. 9.2.1-9.2.6. Wilcy, New York, NY.

Sundberg, J. 1994. The nude (nu) and streaker $\left(\mathrm{nu}^{\mathrm{str}}\right)$ mutations, chromosome 11. In Handbook of mouse mutations with skin and hair abnormalities: Animal models and biomedical tools (ed. J. Sundberg), pp. 379-389. CRC Press, Bar Harbor, ME.

Takahashi, H. and H. Iizuka. 1993. Analysis of the 5'-upstream promoter region of human involucrin gene: Activation by 12-O-tetradecanoylphorbol-13-acetate. I. Invest. Dermatol. 100: $10-15$

Weinberg, W., L. Goodman, C. George, D. Morgan, S. Ledbetter, S.H. Yuspa, and U. Lichti. 1993. The reconstitution of hair follicle development in vivo: Determination of follicle formation, hair growth, and hair quality by dermal cells. $/$. In vest. Dermatol. 100: 229-236.

Welter, J.F. and R.L. Eckert. 1995. Differential expression of the fos and jun family members c-fos, fosB, Fra-1, Fra-2, c-jun, junB and junD during human epidermal keratinocyte differentiation. Oncogene 11: 2681-2687.

Xiong, Y., G. Hannon, H. Zhang, D. Casso, R. Kobayashi, and D. Beach. 1993. p21 is a universal inhibitor of cyclin kinases. Nature 366: 701-704. 


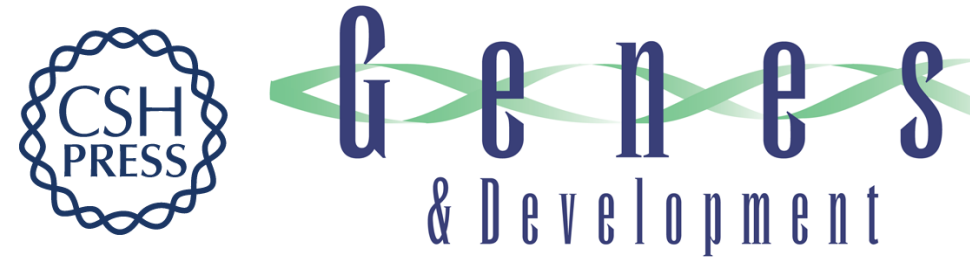

\section{The product of the mouse nude locus, Whn, regulates the balance between epithelial cell growth and differentiation.}

J L Brissette, J Li, J Kamimura, et al.

Genes Dev. 1996, 10:

Access the most recent version at doi:10.1101/gad.10.17.2212

References This article cites 43 articles, 16 of which can be accessed free at:

http://genesdev.cshlp.org/content/10/17/2212.full.html\#ref-list-1

License

Email Alerting

Service

Receive free email alerts when new articles cite this article - sign up in the box at the top right corner of the article or click here.

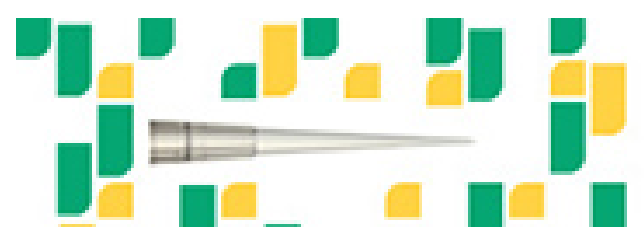

Focused on your science. 